\title{
Sensor Selection for Aircraft Engine Performance Estimation and Gas Path Fault Diagnostics
}

\author{
Paper GT2015-43744
}

\author{
Donald L. Simon \\ NASA Glenn Research Center \\ 21000 Brookpark Road \\ Cleveland, $\mathrm{OH}, 44135$
}

\author{
Aidan W. Rinehart \\ Vantage Partners, LLC \\ 3000 Aerospace Parkway \\ Brook Park, OH 44142
}

ASME Turbo Expo 2015

June 15-19, 2015

Montreal, Canada 


\section{Outline}

- Background - aircraft engine performance estimation and gas path fault diagnostics

- Application-Specific Sensor Selection Metrics

- Kalman filter-based health parameter estimation

- Maximum a posteriori health parameter estimation

- Weighted least squares single fault diagnostic approach

- Linear Turbofan Engine Model Example

- Conclusions 


\section{Background - aircraft engine performance estimation and gas path fault diagnostics}

- Performance Estimation:

- Estimation and trending of gradual performance deterioration due to fouling and erosion of turbomachinery

- Entails the estimation of health parameters such as efficiency and flow capacity scalars, which reflect deterioration in major engine components

- Poses an underdetermined estimation problemmore unknowns than available sensor measurements

- Gas Path Fault Diagnostics:

- Detection and isolation of gas path system faults affecting engine performance such as sensor faults, actuator faults, turbomachinery damage

- Faults are relatively abrupt or rapid in nature

- Single-fault assumption makes the diagnostic problem overdetermined

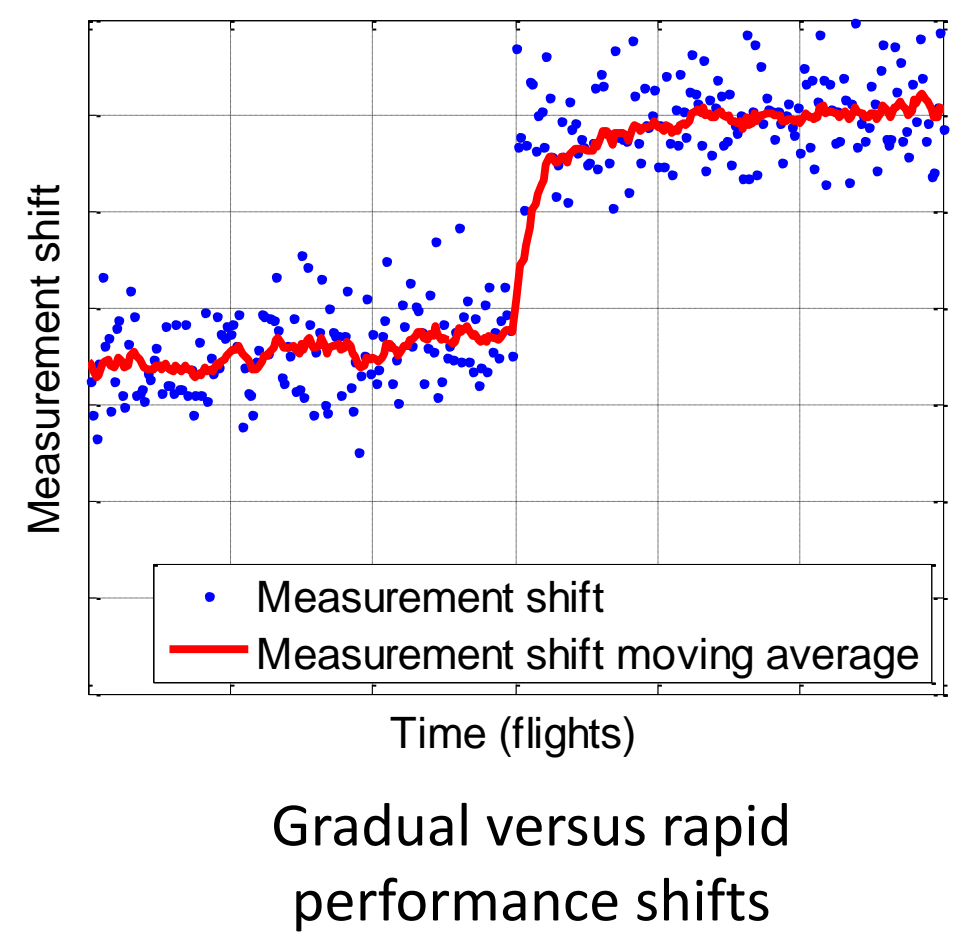




\section{Sensor Selection}

- Problem: In general, additional sensed measurements will improve estimation and diagnostic results, but which sensors are best and how much improvement will they provide?

- Objective: Develop techniques to aid in engine health management sensor selection decisions, tailored to the specific estimation or diagnostic method applied.

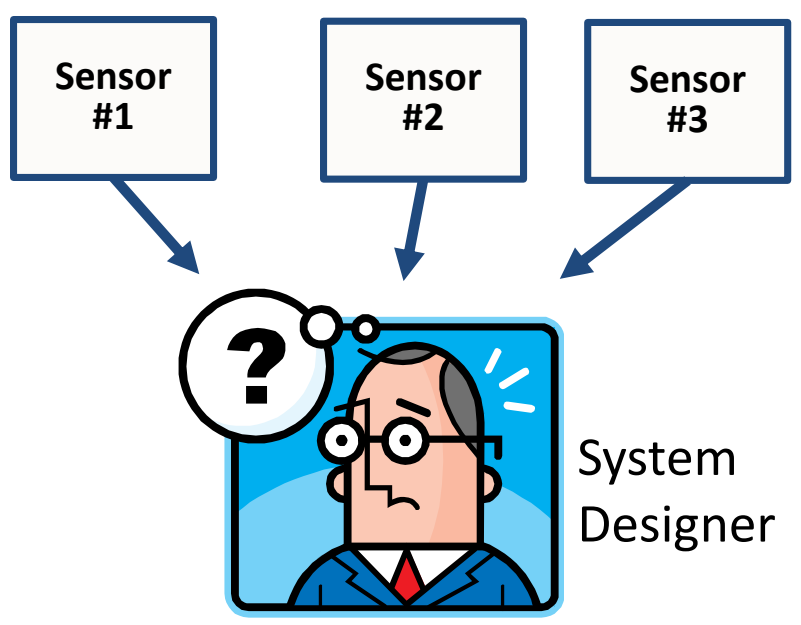

- Approach: Develop analytical metrics based on linear estimation and probability theory to quantify theoretical accuracy enabled by different candidate sensor suites. 


\section{Kalman filter-based performance estimation}

Linear dynamic measurement process:

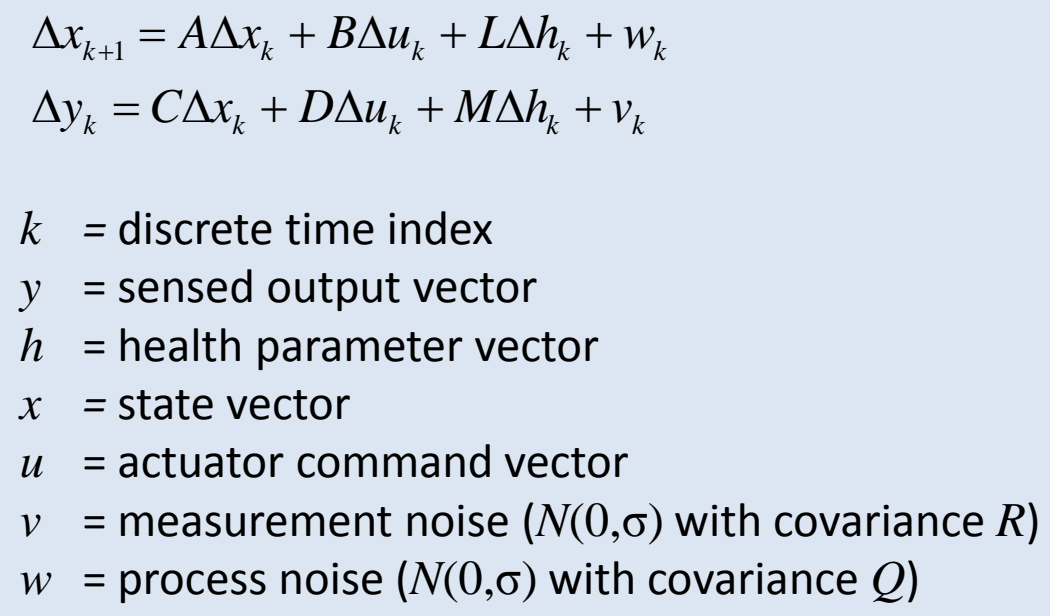

Optimal tuner selection methodology* applied to produce reduced-order system and enable Kalman filter estimation when facing underdetermined estimation problem:

- $\quad$ Define $q=V^{*} h$ where $V^{*}$ is a transformation matrix

- $V^{*}$ is selected through an optimal iterative search to minimize Kalman filter mean squared estimation error in the parameters of interest

- Health parameter estimation: $\hat{h}=V^{*+} \hat{q}$
Sensor selection methodology designed to determine sensor suite that minimizes the mean SSEE

Kalman filter mean sum of squared estimation errors (SSEE) is the sum of the following components:

- Mean squared bias

- Variance

Kalman filter mean squared bias and variance are functions of:

- Linear state-space model

- Choice of $V^{*}$

- Process noise, $Q$

- Health parameter covariance, $P_{h}$

- Available sensor suite and corresponding measurement covariance, $R$ 


\section{Maximum a posteriori (MAP) performance estimation}

Linear steady-state measurement process:

$\Delta y=H \Delta h+v$

$\Delta y=$ residuals in the sensed measurement vector

$H$ = influence coefficient matrix

$\Delta h=$ health parameter vector

$v \quad=$ measurement noise $(N(0, \sigma)$ with covariance $R)$

Maximum a posteriori estimator:

$\Delta \hat{h}=\underbrace{\left(P_{h}^{-1}+H^{T} R^{-1} H\right)^{-1} H^{T} R^{-1}}_{G_{h}} \Delta y$

$\Delta \hat{h}=G_{h} \Delta y$

$P_{h}$ is the health parameter covariance matrix.

Note: Incorporating a priori knowledge through $P_{h}$ allows estimates to be produced when facing underdetermined estimation problems
Sensor selection methodology designed to determine sensor suite that minimizes the mean SSEE

MAP estimation mean sum of squared estimation errors (SSEE) is the sum of the following components:

- Mean squared bias:

$$
\overline{\tilde{h}}^{2}=\operatorname{tr}\left\{\left(G_{h} H-I\right) P_{h}\left(G_{h} H-I\right)^{T}\right\}
$$

- Variance :

$$
\operatorname{var}=\operatorname{tr}\left\{G_{h} R G_{h}^{T}\right\}
$$

MAP mean squared bias and variance are functions of:

- Linear state-space model

- Health parameter covariance, $P_{h}$

- Available sensor suite and corresponding measurement covariance, $R$ 


\section{Weighted Least Squares Single Fault Diagnostic Approach}

Linear steady-state measurement process:

$\Delta \Delta y=H_{f} f+v$

$\Delta \Delta y=$ vector of measurement residuals reflecting recent abrupt shifts in sensor measurements

$H_{f} \quad$ = fault influence coefficient matrix

$f \quad=$ vector of gas path fault magnitudes

$v \quad=$ measurement noise $(N(0, \sigma)$ with covariance $R)$

Fault diagnostics performed via a two-step process:

1) Fault detection performed by monitoring a weighted sum of squared measurements (WSSM):

$$
W S S M=\Delta \Delta y^{\mathrm{T}} R^{-1} \Delta \Delta y
$$

If $W S S M>$ Threshold, $T$, fault declared

2) Single fault isolation performed by comparing known system fault signatures to observed vector of measurement residuals, $\Delta \Delta y$. Fault type that most closed matches observed signature in a weighted least squares sense is isolated as fault.
Estimated fault signatures for each candidate fault type

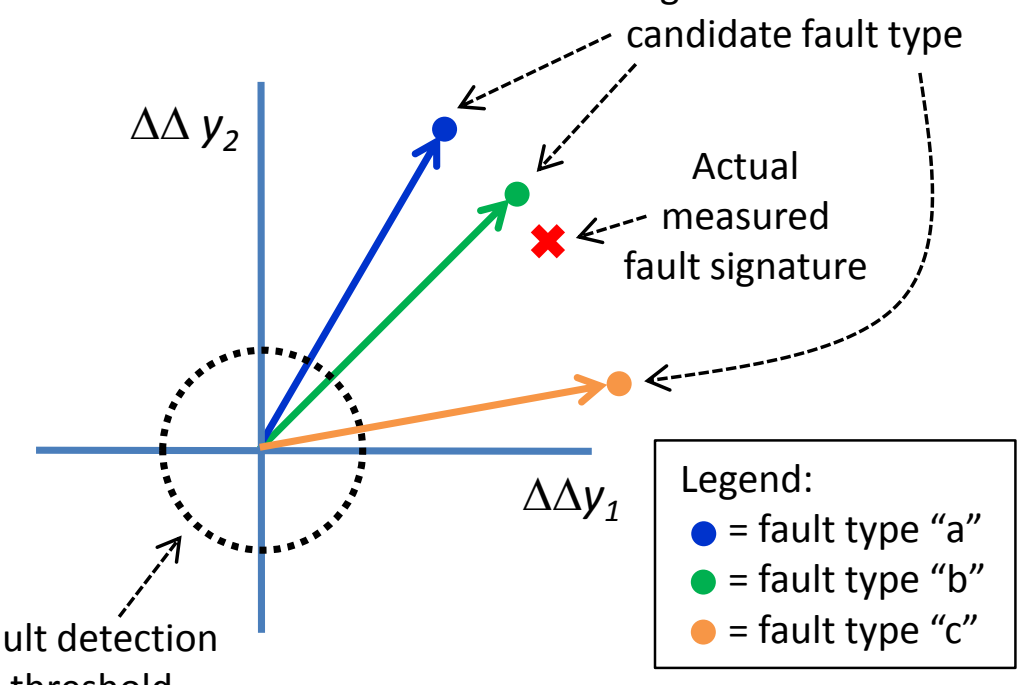
threshold

Illustration of Single-Fault Diagnostic Approach in

Two-Dimensional Measurement Space

- Fault signature, exceeding defined failure threshold, is detected (indicated by red " $x$ ")

- Fault signatures of three system fault types (a, b, and c) are individually compared to measured fault signature

- In this example, fault type " $b$ " will be classified as the fault as it most closely approximates the observed fault signature 


\section{Single Fault Diagnostic Approach (continued)}

Sensor selection methodology determines sensor suite that maximizes the correct classification rate (CCR)

1) WSSM signal fault detection threshold, $T$, is set to yield a common target false positive rate (FPR):

$$
\operatorname{FPR}(T, k)=1-\frac{\gamma\left(\frac{k}{2}, \frac{T}{2}\right)}{\Gamma\left(\frac{k}{2}\right)}
$$

Where:

$T=$ WSSM detection threshold

$k=$ Number of sensors

$\lambda=$ Mean value of the WSSM signal

$\Gamma=$ Gamma function

2) In the presence of a fault, the WSSM signal is distributed as a non-central chi-square distribution, and the true positive rate (TPR) is calculated as:

$$
\operatorname{TPR}(T, k, \lambda)=1-\left(\sum_{j=0}^{\infty} e^{-\lambda / 2} \frac{(\lambda / 2)^{j}}{j !} \frac{\gamma(j+k / 2, T / 2)}{\Gamma(j+k / 2)}\right)
$$

Where:

$\lambda=$ Mean value of WSSM signal in the presence of a fault
3) Applying a two-fault class assumption, the probability of misclassifying fault type "a" as fault type " $b$ " is approximated as:

$$
P M C_{b \mid a}=1-\Phi\left(\frac{1}{2} \cdot D_{M}\right)
$$

Where:

$\Phi=$ Standard normal distribution

$D_{M}=$ Mahalanobis distance

The probability of misclassifying fault type "a" as any other single fault type is:

$$
P M C_{a}=\sum_{\substack{b=1 \\ b \neq a}}^{N} P M C_{b \mid a}
$$

Where:

$N=$ number of fault types

4) Correct classification rate (CCR) for fault type "a" and all fault types is:

$$
\begin{aligned}
& C C R_{a}=T P R_{a} \times\left(1-P M C_{a}\right) \\
& C C R=\sum_{a=1}^{N} \frac{C C R_{a}}{N}
\end{aligned}
$$




\section{Linear Turbofan Engine Model Example}

The sensor selection approaches were applied to a linear point model extracted from the NASA Commercial Modular Aero-Propulsion System Simulation 40k (C-MAPSS40k) high-bypass turbofan engine model.

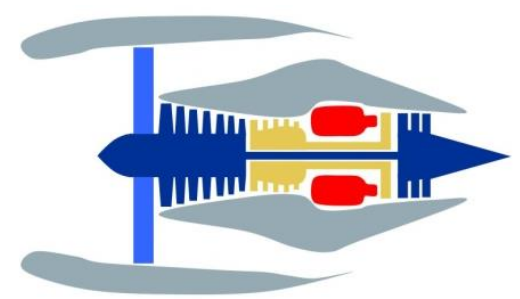

\begin{tabular}{|c|c|c|c|c|}
\hline 7 State variables, $x$ & 3 Actuators, $u$ & 10 Health Parameters, $h$ & \multicolumn{2}{|c|}{6 baseline +4 Optional Sensed outputs, $y$} \\
\hline $\mathrm{Nf}$ - fan speed & Wf - fuel flow & FAN efficiency & $\mathrm{Nf}$ - fan speed & \multirow{6}{*}{$\begin{array}{l}\text { Baseline } \\
\text { Sensors }\end{array}$} \\
\hline $\mathrm{Nc}$ - core speed & VSV - variable stator vane & FAN flow capacity & Nc-core speed & \\
\hline Hs_LPC - LPC metal temp & VBV - variable bleed valve & LPC efficiency & Ps30-HPC exit static press & \\
\hline Hs_HPC - HPC metal temp & & LPC flow capacity & T30 - HPC exit total temp & \\
\hline Hs_burner -burner metal temp & & HPC efficiency & P50 - LPT exit total pressure & \\
\hline Hs_HPT - HPT metal temp & & HPC flow capacity & T50 - LPT exit total temp & \\
\hline \multirow[t]{4}{*}{ Hs_LPT - LPT metal temp } & & HPT efficiency & P14 - Bypass duct total pressure & \multirow{4}{*}{$\begin{array}{l}\text { Additional } \\
\text { (Optional) } \\
\text { Sensors }\end{array}$} \\
\hline & & HPT flow capacity & T14 - Bypass duct total temp & \\
\hline & & LPT efficiency & P25 - HPC inlet total pressure & \\
\hline & & LPT flow capacity & T25 - HPC inlet total temp. & \\
\hline
\end{tabular}

Objective: assess the estimation and diagnostic improvements that can be gained by adding sensors individually or in combination to the baseline sensor suite 


\section{Sensor Selection for Health Parameter Estimation}

Health Parameter Estimation:

- Objective is to minimize sum of squared estimation errors (SSEE) across all 10 health parameters

- Health parameters are assumed to exhibit simultaneous, uncorrelated, normally distributed random variations with a standard deviation of $\pm 2 \%$

- Health parameter covariance matrix, $P_{h}$, is a $10 \times 10$ diagonal matrix with elements of 4.0 along the diagonal.

\section{C-MAPSS40k Health Parameters}

\begin{tabular}{ccl}
\hline \hline \multicolumn{3}{c}{ Health parameters } \\
\hline 1 & $\eta_{\text {FAN }}$ & Fan efficiency \\
2 & V FAN & Fan flow capacity \\
3 & $\eta_{\text {LPC }}$ & Low pressure compressor (LPC) efficiency \\
4 & $Y_{\text {LPC }}$ & Low pressure compressor (LPC) flow capacity \\
5 & $\eta_{\text {HPC }}$ & High pressure compressor (HPC) efficiency \\
6 & $Y_{\text {HPC }}$ & High pressure compressor (HPC) flow capacity \\
7 & $\eta_{\text {HPT }}$ & High pressure turbine (HPT) efficiency \\
8 & $Y_{\text {HPT }}$ & High pressure turbine (HPT) flow capacity \\
9 & $\eta_{\text {LPT }}$ & Low pressure turbine (LPT) efficiency \\
10 & $Y_{\text {LPT }}$ & Low pressure turbine (LPT) flow capacity \\
\hline \hline
\end{tabular}

Analytical techniques applied to predict theoretical SSEE for each candidate sensor suite

Monte Carlo simulations conducted to validate theoretical predictions

- Uses C-MAPSS40k linear point model

- Health parameters randomly assigned according to health parameter covariance matrix, $\boldsymbol{P}_{\boldsymbol{h}}$

- Random sensor measurement noise added in accordance with sensor measurement covariance matrix, $\boldsymbol{R}$

- Kalman Filter Estimator - 200 trials, each 30 seconds in duration

- MAP Estimator - 400,000 trials, each a single sample in time 


\section{Sensor Selection Results for}

\section{Health Parameter Estimation}

\section{Kalman Filter Estimator Results}

\begin{tabular}{|c|c|c|c|c|c|c|}
\hline \multirow{2}{*}{ 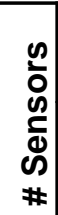 } & \multicolumn{4}{|c|}{$\begin{array}{c}\text { sensors added } \\
\text { to baseline }\end{array}$} & \multicolumn{2}{|c|}{ Sum of Squared Estimation Errors } \\
\hline & $\frac{\dot{T}}{\alpha}$ & $\stackrel{\nabla}{F}$ & ฉి & $\stackrel{\sim}{\sim}$ & Theoretical & $\begin{array}{l}\text { Monte Carlo } \\
\text { Simulation }\end{array}$ \\
\hline 6 & & & & & 17.21 & 17.35 \\
\hline 7 & $\mathbf{x}$ & & & & 13.66 & 13.94 \\
\hline 7 & & $\mathbf{x}$ & & & 13.81 & 14.44 \\
\hline 7 & & & $\mathbf{x}$ & & 12.83 & 13.19 \\
\hline 7 & & & & $x$ & 12.58 & 12.90 \\
\hline 8 & $\mathbf{x}$ & $\mathbf{x}$ & & & 22.45 & 23.74 \\
\hline 8 & $\mathbf{x}$ & & $\mathbf{x}$ & & 9.14 & 9.94 \\
\hline 8 & $\mathbf{x}$ & & & $\mathbf{x}$ & 8.78 & 9.60 \\
\hline 8 & & $\mathbf{x}$ & $\mathbf{x}$ & & 10.44 & 11.47 \\
\hline 8 & & $\mathbf{x}$ & & $\mathbf{x}$ & 9.27 & 9.84 \\
\hline 8 & & & $x$ & $x$ & 8.60 & 8.90 \\
\hline 9 & $\mathbf{x}$ & $\mathbf{x}$ & $x$ & & 10.07 & 11.66 \\
\hline 9 & $x$ & $x$ & & $\mathbf{x}$ & 6.13 & 7.29 \\
\hline 9 & $x$ & & $x$ & $x$ & 4.79 & 5.45 \\
\hline 9 & & $x$ & $x$ & $x$ & 4.95 & 5.75 \\
\hline 10 & $x$ & $\mathbf{x}$ & $\mathbf{x}$ & $\mathbf{x}$ & 4.47 & 4.98 \\
\hline
\end{tabular}

MAP Estimator Results

\begin{tabular}{|c|c|c|c|c|c|c|}
\hline \multirow{2}{*}{ 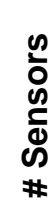 } & \multicolumn{4}{|c|}{$\begin{array}{l}\text { sensors added } \\
\text { to baseline }\end{array}$} & \multicolumn{2}{|c|}{ Sum of Squared Estimation Errors } \\
\hline & $\frac{ \pm}{a}$ & $\stackrel{ \pm}{F}$ & $\stackrel{\sim}{ณ}$ & $\stackrel{\mathscr{N}}{\sim}$ & Theoretical & $\begin{array}{c}\text { Monte Carlo } \\
\text { Simulation }\end{array}$ \\
\hline 6 & & & & & 16.35 & 16.36 \\
\hline 7 & $\mathbf{x}$ & & & & 12.86 & 12.86 \\
\hline 7 & & $\mathbf{x}$ & & & 14.54 & 14.55 \\
\hline 7 & & & $\mathbf{x}$ & & 12.36 & 12.37 \\
\hline 7 & & & & $\mathrm{x}$ & 12.36 & 12.36 \\
\hline 8 & $\mathbf{x}$ & $\mathbf{x}$ & & & 12.38 & 12.38 \\
\hline 8 & $\mathbf{x}$ & & $\mathbf{x}$ & & 8.87 & 8.86 \\
\hline 8 & $\mathbf{x}$ & & & $\mathbf{x}$ & 8.87 & 8.86 \\
\hline 8 & & $\mathbf{x}$ & $\mathbf{x}$ & & 10.55 & 10.55 \\
\hline 8 & & $\mathbf{x}$ & & $\mathbf{x}$ & 10.55 & 10.54 \\
\hline 8 & & & $\mathbf{x}$ & $\mathbf{x}$ & 8.40 & 8.41 \\
\hline 9 & $\mathbf{x}$ & $\mathbf{x}$ & $\mathbf{x}$ & & 8.39 & 8.38 \\
\hline 9 & $\mathbf{x}$ & $\mathbf{x}$ & & $\mathbf{x}$ & 8.39 & 8.38 \\
\hline 9 & $x$ & & $x$ & $x$ & 4.91 & 4.91 \\
\hline 9 & & $\mathbf{x}$ & $x$ & $\mathbf{x}$ & 6.59 & 6.60 \\
\hline 10 & $\mathbf{x}$ & $\mathbf{x}$ & $\mathbf{x}$ & $\mathbf{x}$ & 4.43 & 4.43 \\
\hline
\end{tabular}

Sensor Selection Results (Kalman filter and MAP estimator select the same sensors):

- Baseline + 1 sensor, choose: T25

- Baseline + 2 sensors, choose: T25 and P25

- Baseline + 3 sensors, choose: T25, P25, and P14 


\section{Sensor Selection for Gas Path Fault Diagnostics}

\section{Gas Path Fault Diagnostics:}

- Objective is to maximize the correct classification rate (CCR) across all 8 gas path faults

- Each fault considered to occur in isolation, and to be of equal criticality and probability of occurrence

\section{Gas Path Fault Types}

\begin{tabular}{cll}
\hline $\begin{array}{c}\text { Fault } \\
\text { ID }\end{array}$ & $\begin{array}{l}\text { Fault } \\
\text { type }\end{array}$ & $\begin{array}{l}\text { Health parameters and } \\
\text { actuator biases }\end{array}$ \\
\hline 1 & Fan fault & $\eta_{\text {FAN }}=-1 \%, Y_{\text {FAN }}=-2 \%$ \\
2 & LPC fault & $\eta_{\text {LPC }}=-1 \%, Y_{\text {LPC }}=-2 \%$ \\
3 & HPC fault & $\eta_{\text {HPC }}=-1 \%, Y_{\text {HPC }}=-2 \%$ \\
4 & HPT fault & $\eta_{\text {HPT }}=-2 \%, Y_{H P T}=+1 \%$ \\
5 & LPT fault & $\eta_{\text {LPT }}=-2 \%, Y_{\text {LPT }}=+1 \%$ \\
6 & Wf bias & Wf bias $=-2 \%$ \\
7 & VSV bias & VSV bias $=-1$ degree stroke \\
8 & VBV bias & VBV bias $=+20 \%$ \\
\hline \hline
\end{tabular}

Analytical techniques applied to predict theoretical CCR for each candidate sensor suite

Monte Carlo simulations conducted to validate theoretical predictions

- Uses C-MAPSS40k linear fault influence coefficient matrix

- Random sensor measurement noise added in accordance with sensor measurement covariance matrix, $R$

- Monte Carlo simulation study consisted of 80,000 no fault cases and 10,000 cases for each of the 8 gas path fault types

- Detection threshold set to achieve theoretical false positive rate of 0.01 (1\%) 


\section{Sensor Selection Results for Gas Path Diagnostics}

\section{Gas Path Diagnostic Results}

\begin{tabular}{|c|c|c|c|c|c|c|c|c|}
\hline \multirow{2}{*}{ 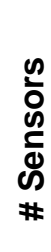 } & \multicolumn{4}{|c|}{$\begin{array}{l}\text { sensors } \\
\text { added to } \\
\text { baseline }\end{array}$} & \multicolumn{2}{|c|}{$\begin{array}{l}\text { False Positive } \\
\text { Rate (\%) }\end{array}$} & \multicolumn{2}{|c|}{$\begin{array}{c}\text { Correct Classification } \\
\text { Rate (\%) }\end{array}$} \\
\hline & $\frac{\nabla}{a}$ & $\frac{Z}{F}$ & ณ & $\stackrel{\text { ก }}{\leftarrow}$ & Theoretical & $\begin{array}{l}\text { Monte Carlo } \\
\text { Simulation }\end{array}$ & Theoretical & $\begin{array}{c}\text { Monte Carlo } \\
\text { Simulation }\end{array}$ \\
\hline 6 & & & & & 1.00 & 1.04 & 84.60 & 88.40 \\
\hline 7 & $x$ & & & & 1.00 & 1.02 & 85.51 & 89.06 \\
\hline 7 & & $x$ & & & 1.00 & 0.99 & 85.84 & 89.18 \\
\hline 7 & & & $x$ & & 1.00 & 1.00 & 88.98 & 90.39 \\
\hline 7 & & & & $x$ & 1.00 & 1.07 & 91.58 & 92.59 \\
\hline 8 & $x$ & $x$ & & & 1.00 & 1.03 & 86.29 & 89.47 \\
\hline 8 & $\mathbf{x}$ & & $x$ & & 1.00 & 1.03 & 89.73 & 91.11 \\
\hline 8 & $\mathbf{x}$ & & & $\mathbf{x}$ & 1.00 & 1.05 & 92.28 & 93.23 \\
\hline 8 & & $x$ & $\mathbf{x}$ & & 1.00 & 1.02 & 89.97 & 91.19 \\
\hline 8 & & $x$ & & $x$ & 1.00 & 1.02 & 92.42 & 93.30 \\
\hline 8 & & & $x$ & $\mathbf{x}$ & 1.00 & 1.01 & 92.04 & 92.57 \\
\hline 9 & $\mathbf{x}$ & $x$ & $x$ & & 1.00 & 1.02 & 90.28 & 91.48 \\
\hline 9 & $x$ & $x$ & & $x$ & 1.00 & 1.02 & 92.69 & 93.55 \\
\hline 9 & $x$ & & $x$ & $\mathbf{x}$ & 1.00 & 1.01 & 92.70 & 93.29 \\
\hline 9 & & $x$ & $x$ & $x$ & 1.00 & 1.02 & 92.83 & 93.32 \\
\hline 10 & $\mathbf{x}$ & $\mathbf{x}$ & $x$ & $\mathbf{x}$ & 1.00 & 1.00 & 93.07 & 93.57 \\
\hline
\end{tabular}

Monte Carlo results

- Confirmed theoretical target false positive rate of $1 \%$

- Theoretical correct classification rate (CCR) found to under-predict Monte Carlo CCR. This is attributed to the 2 fault class simplifying assumption made in calculating the theoretical CCR.

Sensor Selection Choices:

- Baseline + 1 sensor, choose: T25

- Baseline +2 sensors, choose: T25 and T14

- Baseline + 3 sensors:

○ Theoretical choose: T25, T14, and P25

o Monte Carlo choose: T25, T14 and P14 


\section{Conclusions}

- Sensor selection methods introduced in this paper were found to perform well in identifying optimal sensor suites

- Results are application-specific to engine model considered and measurement noise, health parameter variations, and fault types assumed

- Kalman filter and MAP estimator based sensor-selection methods found to yield good agreement between theoretical predictions and simulation results. Also found to yield same sensor selection choices.

- Weighted Least Squares Single Fault Diagnostic sensor selection methods found to slightly under-predict correct classification rate

- Follow-on recommendations

- Incorporate other factors of merit such as sensor life cycle cost (cost, weight, reliability, etc.) and criticality of different fault types

- Extend to additional operating points beyond single linear point analysis 


\section{Acknowledgments}

- This work was conducted under the NASA Aviation Safety Program, Vehicle Systems Safety Technologies Project and the NASA Transformative Aeronautics Concept Program, Convergent Aeronautics Solutions Project

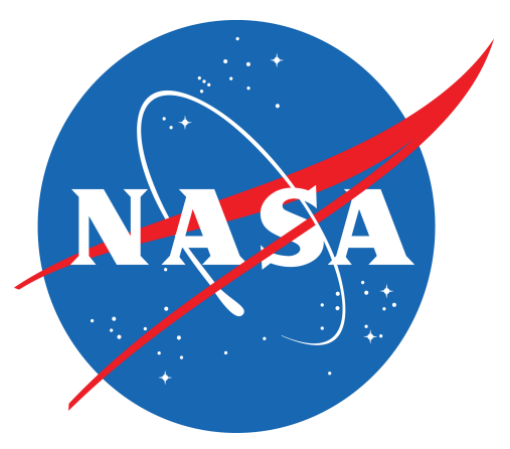




\section{Backup Slides}




\section{Linear Turbofan Engine Model Example}

\section{Sensor Selection Approach:}

- Optional sensors are evaluated for estimation accuracy or diagnostic improvement they provide if added individually or in combination to baseline sensor suite.

- Given $n$ sensors to choose from, and a target number, $k$, of additional sensors, the total number of sensor suite combinations will be:

$$
\left(\begin{array}{l}
n \\
k
\end{array}\right)=\frac{n !}{k !(n-k) !}
$$

- Thus, the number of sensor combinations when adding 1, 2, 3, or 4 sensors to the baseline 6 sensors are:

- Baseline sensors

- Baseline +1 sensor

- Baseline +2 sensors

- Baseline +3 sensors

- Baseline +4 sensors
1 combination

4 combinations

6 combinations

4 combinations

1 combination

- Analytical metrics are applied to calculate theoretical performance for each sensor suite

- Monte Carlo simulation analysis is then conducted to verify theoretical predictions 


\section{Performance estimation and gas path fault diagnostic methods considered in this study}

- Performance Estimation Methods:

- Kalman filter estimation

- Applied in dynamic, streaming (continuous) engine measurement process

- Underdetermined estimation addressed by combining health parameters into a reduced set of optimal tuners

- Maximum a posteriori estimation

- Applied in steady-state measurement process as available through "snapshot" measurements

- Underdetermined estimation addressed by leveraging a priori knowledge regarding health parameter covariance

- Gas Path Fault Diagnostic Method:

- Weighted-least squares single fault diagnostic approach

- Fault detection performed by monitoring for abrupt shifts in measurements

- Fault isolation performed by identifying the known fault signature that most closely matches observed measurement signature in multi-parameter measurement space in a weighted-least squares approach

Sensor selection methods tailored to given estimation/diagnostic method 